\title{
Control of bimanual rhythmic movements: trading efficiency for robustness depending on the context
}

\author{
Renaud Ronsse $\cdot$ Jean-Louis Thonnard $\cdot$ \\ Philippe Lefèvre $\cdot$ Rodolphe Sepulchre
}

Received: 20 September 2007/ Accepted: 17 January 2008/Published online: 14 February 2008

(C) Springer-Verlag 2008

\begin{abstract}
This paper investigates how the efficiency and robustness of a skilled rhythmic task compete against each other in the control of a bimanual movement. Human subjects juggled a puck in $2 \mathrm{D}$ through impacts with two metallic arms, requiring rhythmic bimanual actuation. The arms kinematics were only constrained by the position, velocity and time of impacts while the rest of the trajectory did not influence the movement of the puck. In order to expose the task robustness, we manipulated the task context in two distinct manners: the task tempo was assigned at four different values (hence manipulating the time available to plan and execute each impact movement individually); and vision was withdrawn during half of the trials (hence reducing the sensory inflows). We show that when the tempo was fast, the actuation was rhythmic (no pause in the trajectory) while at slow tempo, the actuation was discrete (with pause intervals between individual
\end{abstract}

R. Ronsse · R. Sepulchre

Department of Electrical Engineering and Computer Science (Montefiore Institute), Université de Liège, Grande Traverse 10 (B28), 4000 Liège, Belgium

J.-L. Thonnard

Unité de Réadaptation et de Médecine Physique,

Université catholique de Louvain, Avenue Mounier 53,

1200 Bruxelles, Belgium

P. Lefèvre $(\bowtie)$

Centre for Systems Engineering and Applied Mechanics

(CESAME), Université catholique de Louvain, Avenue Georges

Lemaître 4, 1348 Louvain-la-Neuve, Belgium

e-mail: Philippe.Lefevre@UCLouvain.be

P. Lefèvre

Laboratory of Neurophysiology, Université catholique de

Louvain, Avenue Hippocrate 54, 1200 Bruxelles, Belgium movements). Moreover, the withdrawal of visual information encouraged the rhythmic behavior at the four tested tempi. The discrete versus rhythmic behavior give different answers to the efficiency/robustness trade-off: discrete movements result in energy efficient movements, while rhythmic movements impact the puck with negative acceleration, a property preserving robustness. Moreover, we report that in all conditions the impact velocity of the arms was negatively correlated with the energy of the puck. This correlation tended to stabilize the task and was influenced by vision, revealing again different control strategies. In conclusion, this task involves different modes of control that balance efficiency and robustness, depending on the context.

Keywords Rhythmic movements · Discrete movements · Impact juggling - Sensorimotor loop - Robust control · Energy-optimal control

\section{Introduction}

The computational approach is widely used in the motor control literature, providing a unified framework to study motor planning, control, estimation, prediction and learning (Jordan and Wolpert 1999; Wolpert and Ghahramani 2000; Bays and Wolpert 2007). Such concepts are inherited from well established principles from system-theoretic engineering, and their exploitation in motor control remarkably parallels with analogous designs in robotics (Schaal and Schweighofer 2005). As usually done in the context of engineering designs, a conceptual diagram of motor control can be elaborated as an assembling of different functional compartments, referring to the various computational steps throughout the motor pathway. The 
highest level refers to the planning of the trajectory, i.e., the computation of the desired trajectory. Downstream from planning, the movement control refers to mechanisms used to compute the motor command in order to maintain the actual trajectory close to the desired one. The processing of sensory inflows may obviously influence the motor command (control), but also necessitates to recompute the reference trajectory (planning). Note that the optimal control theory has been recently used to provide an elegant framework to capture planning and control into a single executive system (Todorov and Jordan 2002; Scott 2004; Todorov 2004, 2006; Bays and Wolpert 2007; Liu and Todorov 2007).

The present paper aims at shedding light on a particular computational aspect that is ubiquitous in control theory: the trade-off between efficiency and robustness (often referred as the performance/robustness trade-off in control literature, see, e.g., Boulet and Duan 2007). Efficiency quantifies how well a system performs (e.g., through the minimization of a cost function); and robustness quantifies how the stability is maintained despite uncertainties and perturbations. For example, robustness can be quantified by the noise level and the computational delay which are tolerated before losing stability. Very often, efficiency and robustness compete against each other, in the sense thatfor a fixed controller structure-the efficiency can only be increased above a certain threshold by "sacrificing" the robustness. Many textbooks in control theory (see, e.g., Franklin et al. 2005; Åström and Murray 2008) provide caveat examples of closed-loop designs performing excellently, but fragile to a slight change in any parameter. Different requirements in efficiency and/or robustness can therefore strongly influence the design of the control structure, as well as the processing of sensory information. Evidence for robust control has been shown in motor execution, as demonstrated, e.g., for arm reaching (see, e.g., Karniel and Inbar 2000; Liu and Todorov 2007) or saccade execution (see, e.g., Harris and Wolpert 1998, 2006). The present paper illustrates how the trade-off between efficiency and robustness influences the movement execution in the particular context of a bimanual rhythmic movement.

The task studied in this paper is an original simplified juggling task (2D). It is a bimanual version of the ball + racket task, extensively studied in the motor control literature (see, e.g., Schaal et al. 1996; Sternad 1999; Sternad et al. 2001a, b; deRugy et al. 2003; Wei et al. 2007). Our task requires the stabilization of a puck trajectory, through periodic impacts with two metallic arms which are actuated by the subject (see Fig. 1). In this task, the periodic movement of the puck forces the arms to be rhythmically actuated, but not necessarily in a sustained (or continuous) manner: only the arms

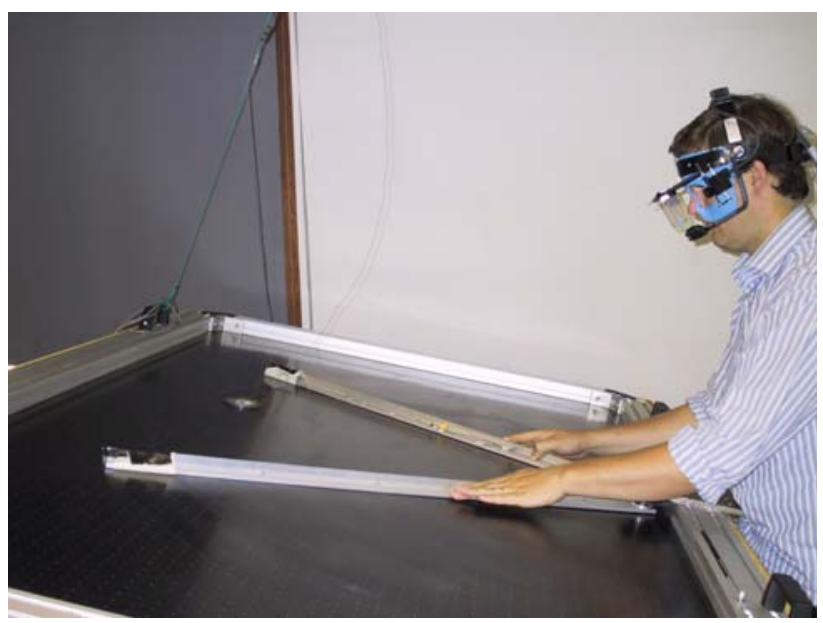

Fig. 1 Experimental setup

kinematics at impacts influence the course of the puck. Such a bimanual movement is constrained by intrinsic coordination rules which attract both arms into stable actuation regimes, such as in-phase and anti-phase (see, e.g., Kelso et al. 1979; Kelso 1995; Swinnen 2002). While in-phase actuation does not make sense in the present task, anti-phase actuation of the arms is the intuitively stable control regime in which phase locking is a primary feature. However, the arms could be also actuated one after the other, in a relatively discrete and decoupled manner, hence relaxing the phase locking between both arms and potentially allowing some dwell intervals between individual movements. While these two strategies are actually rhythmic (since the arms adopt a periodic pattern in both cases), we rely on the terminology proposed by Hogan and Sternad (2007) to differentiate the two types of movements in the rest of the paper: the sustained mode is referred to as "rhythmic", since the arms actuation was sustained with short rest intervals; and the intermittent mode is referred to as "discrete", since the individual impacts are separated by longer rest intervals.

One advantage of the present juggling task is that it is amenable to both mathematical analysis and behavioral experiments (Ronsse et al. 2008). Initially, in Ronsse et al. (2006), we have established that purely sensorless control of this task is theoretically possible. However, this sensorless control is not robust and was hard to validate in robotics experiments. In Ronsse et al. (2007), we showed both theoretically and experimentally that the robustness of the control is significantly enhanced with the help of a cheap source of sensory feedback: the impact times. This discrete, event-related, source of feedback significantly improved the experimental stabilization of periodic juggling patterns. However, robustness remained a critical issue and we have particularly shown that an essential 
feature of this feedback controller was to impact the puck with negative acceleration of the arm, exploiting the sensorless dynamical properties (Schaal et al. 1996).

The aim of the present study is to investigate the human behavior in the light of these theoretical findings. First, we investigated how the withdrawal of vision modifies the task control. The absence of vision should increase the uncertainties in the task, since visual information is very important to measure some state variables (e.g., the puck energy); and to predict the future motion of the puck, such as the forthcoming impact time (Land and McLeod 2000), which is a fundamental control parameter (Ronsse et al. 2007). Second and in parallel, we asked the subjects to perform the task at different tempi, hereby manipulating the time available to execute each impact movement individually. Indeed, increasing the tempo also decreases the time to plan and execute each movement, assuming that the absolute time delay-inherent to the sensorimotor loop-remains constant across conditions. Different tempi can be easily achieved by the subjects, through a proper tuning of the average aperture between the arms (the smaller the aperture, the faster the tempo). In sum, we investigate to what extent maintaining the robustness at a satisfactory level, despite uncertainties, affects the efficiency of the actuation strategy. It is predicted that the conditions in which the task robustness is the most exposed (without vision, or at the fastest tempi), acceleration should be more negative, while the priority is given to maximize the control efficiency in the other conditions. The proposed estimation of the task efficiency is the average energy of the arms, since it can be reasonably hypothesized that the minimization of the energy cost is a key feature of skilled movement control. Previous investigations in the optimal control framework have indeed established that many typical movements result from an attempt to minimize the control cost (Nakano et al. 1999; Todorov and Jordan 2002; Todorov 2004; Liu and Todorov

$400 \mathrm{~ms}$

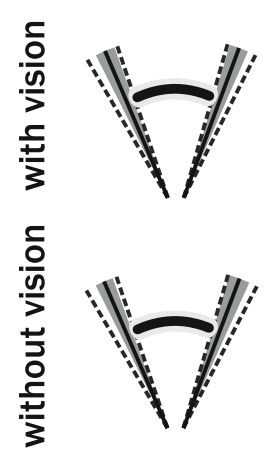

$600 \mathrm{~ms}$
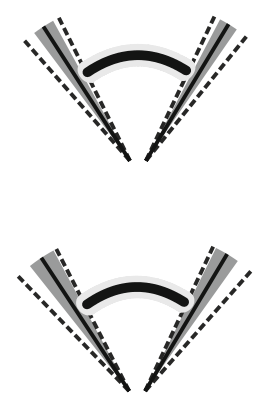

$800 \mathrm{~ms}$
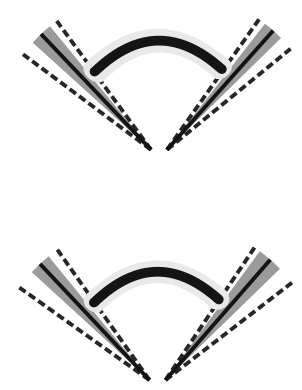

2007), which can be considered as an approximate of the effector energy cost as well.

Given the parallel between the robotics investigations proposed in Ronsse et al. (2006, 2007) and the present contribution, our simplified juggling experiment is thus suited to address questions in robotics and motor control in parallel (Schaal and Schweighofer 2005), in the particular context of bimanual rhythmic movements.

\section{Materials and methods}

Task and experimental setup description

Nine healthy human subjects (4 female, 5 male, 2328 years old) volunteered to perform a bimanual, impact juggling task. Two subjects were left-handed, seven were right-handed (Edinburgh Handedness Test). One subject is the first author of the paper while the others were naive regarding the goals of the experiment. They provided informed written consent, and reported no history of neurological or musculoskeletal disorder. All had normal vision, either natural or corrected. All the procedures conducted were approved by the local ethics committee, in compliance with the Helsinki declaration.

The subjects stood in front of an air-hockey table that was tilted $12.5^{\circ}$ w.r.t. the ground, and they actuated two independent metallic arms that were free to rotate on the table around their lower extremity (see Fig. 1). The two points of rotation were mounted close to each other, on the table frame. The subjects had to rotate the metallic arms in order to impact a plastic puck (72 $\mathrm{mm}$ diameter) back and forth. The subjects were instructed to stabilize the so-called "period-one" motion of the puck: a single parabola between the arms [see Fig. 2 and Ronsse et al. (2006) for an extended description of the setup].

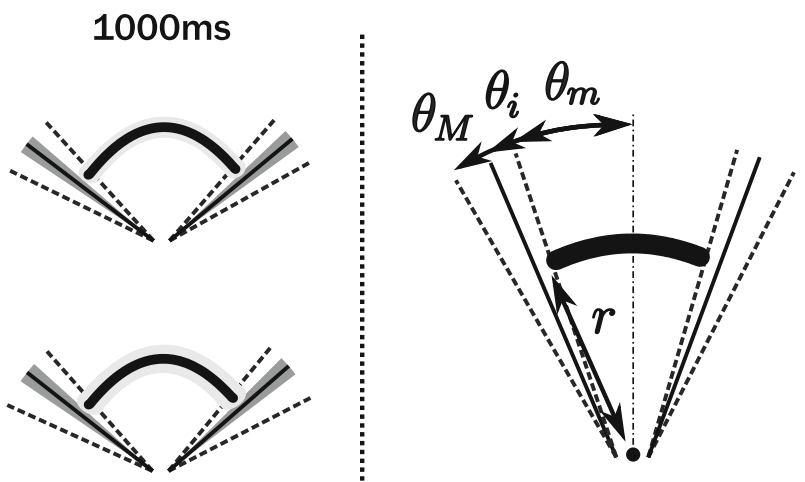

Fig. 2 Average trajectories of the puck for the four tempi and the two vision conditions (averaged across sessions and subjects, black parabolas). As illustrated in the right panel, the solid black lines represent the average position of the arms at impact $\theta_{i}$ (the arms rotate around their lower extremity). The dashed dark gray lines represent the average of the smallest $\left(\theta_{m}\right)$ and the largest $\left(\theta_{M}\right)$ angular position of the arms during the cycle. $r$ denotes the radial position of the puck at impact. The shaded areas correspond to the standard deviations of the mean impact positions and the mean puck trajectories. The numerical values are given in Table 1, pooled for both arms 
The task tempo was assigned by a metronome, that beeped either at $T=400,600,800$ or $1,000 \mathrm{~ms}$ intervals. The subjects were instructed to impact the puck such that the time interval between two successive impacts equaled the metronome tempo. Consequently the tempo $T$ represents the half-period of the steady-state periodic pattern of each arm. At each tempo, the subjects were asked to perform the juggling task for $2 \mathrm{~min}$ in normal visual condition, followed by 2 min with closed eyes. These $2 \times 2$ min blocks were repeated six times per session: one at $T=400 \mathrm{~ms}$, one at $T=600 \mathrm{~ms}$, two at $T=800 \mathrm{~ms}$, and two at $T=1,000 \mathrm{~ms}$. This generated $120 \mathrm{~s} / 0.4 \mathrm{~s}=300$ beeps at $T=400 \mathrm{~ms}$; $120 \mathrm{~s} / 0.6 \mathrm{~s}=200$ beeps at $T=600 \mathrm{~ms} ; 2 \times 120 \mathrm{~s} / 0.8 \mathrm{~s}=$ 300 beeps at $T=800 \mathrm{~ms}$; and $2 \times 120 \mathrm{~s} / 1 \mathrm{~s}=240$ beeps at $T=1,000 \mathrm{~ms}$ per subject $\times$ vision condition $\times$ session, and then potentially the same number of impacts. The succession of tempi was randomly generated before each session. Each of the nine subjects completed four sessions collected on three or four different days, depending on the subject. They were authorized to acquaint themselves with the task during $15 \mathrm{~min}$ before the first session.

A simplified model of this task has been studied in Ronsse et al. (2006, 2007) for robotics applications. This model is based on the combination of a parabolic trajectory during flight time, and an impact rule for the puck velocity. The impact rule assumes that the normal component of the puck velocity $v_{n}$-relative to the arm velocity at impact $r \dot{\theta}_{i}$, i.e., the product between the radial position of impact $r$ and the arm angular velocity at impact $\dot{\theta}_{i}$-is reversed at impact and multiplied by a coefficient of restitution $e$ :

$$
\begin{aligned}
& v_{n}^{+}-r \dot{\theta}_{i}=-e\left(v_{n}^{-}-r \dot{\theta}_{i}\right) \\
& \Leftrightarrow v_{n}^{+}=-e v_{n}^{-}+(1+e) r \dot{\theta}_{i}
\end{aligned}
$$

where the - and + superscripts denote the normal velocity before and after the impact, respectively. Equation 1 properly captures that the post-impact velocity of the puck $\left(v_{n}^{+}\right)$is controlled via the arm velocity at impact $\dot{\theta}_{i}$. The puck energy (per unit of mass) equals $\xi=0.5 v_{r}^{2}+0.5 v_{n}^{2}+g r$, where $v_{r}$ is the radial component of the puck velocity and $g$ the constant of gravity. Thus Eq. 1 also captures how the puck energy is updated through impacts (via the normal velocity $v_{n}$ ) by tuning the impact velocity $\dot{\theta}_{i}$. Steady-state analysis of the model revealed that the tempo of the task corresponds to one steady-state energy level of the puck, for a fixed angular position of impacts $\overline{\theta_{i}}$ (Ronsse et al. 2006):

$\xi=\frac{3+\tan ^{2} \overline{\theta_{i}}}{8 \tan ^{2} \overline{\theta_{i}}} T^{2} g^{2}$.

The coefficient of restitution of the arms-i.e., the ratio between the absolute value of the puck velocity after and before an impact with one arm at rest $\left(\dot{\theta}_{i}=0\right.$ in Eq. 1)was estimated at $e=0.49$.
A Chronos eye tracker (CHRONOS VISION GmbH, Berlin, Germany), which is based on high-frame rate CMOS sensors (Clarke et al. 2002), was used to monitor the correct closing of the eyes when instructed. The 3D positions of infrared light-emitting diodes (IREDs) at the top of the arms and at the center of the puck were measured using an OptoTrak 3020 system (Northern Digital, Ontario, Canada). The OptoTrak was mounted on the ceiling about $3 \mathrm{~m}$ in front of the subject. The positions of the IREDs were rotated to be expressed in a coordinate system with two axes parallel to the air-hockey table frame, and the third one pointing upward, and centered between the rotation points of the arms. The position of each IRED was sampled at $200 \mathrm{~Hz}$ with a resolution of about $0.1 \mathrm{~mm}$ within the working environment. Each block was executed over a period of $120 \mathrm{~s}$, and its data recorded in separate files.

\section{Data analysis}

Digital processing of the raw data was performed with Matlab (the MathWorks Inc., Natick, MA, USA). The arms IREDs were filtered at $10 \mathrm{~Hz}$ by a zero-phase digital filter (autoregressive, forward and backward). The puck trajectories were not filtered because the puck velocity was discontinuous at impact: these discontinuity points identified the impact times. Velocities and accelerations of the puck and the two arms were computed from position signals by means of a central difference algorithm (i.e., with no time lag).

The flight time is defined as the time between two successive impacts. More precisely, the flight time corresponding to impact $k$ is $\Delta t=t[k]-t[k-1]$, where $t[k]$ is the time of impact $k$. Similarly, an arm cycle corresponds to the time interval between two successive impacts on the same arm, that is $t[k]-t[k-2]$.

This paper does not focus on transient or initialization phases. Since the subjects did not maintain steady state cycles during the whole blocks, we kept only the steadystate cycles in the database, i.e. the cycles corresponding to $\Delta t \in[0.2 T, 2 T]$ and impacting the arms alternatively. Furthermore, we kept only the impact runs containing at least four successive impacts, and we removed the two first and the last one. The images of the eye tracker were used to verify that the subjects followed the instruction to close their eyes during the blocks without vision. In the data reported here and corresponding to the "without vision" condition, we kept only the impacts for which we can certify that the eyes were actually closed during at least $80 \%$ of the corresponding puck flight time. Other impacts were not analyzed. After this complete selection, the database contained 28,176 pairs of flights and impacts 
distributed as follows: 6,101 impacts at $T=400 \mathrm{~ms}$ with vision (i.e., $56 \%$ of the metronome beats in the corresponding condition), 2,834 impacts at $T=400 \mathrm{~ms}$ without vision $(26 \%), 4,487$ impacts at $T=600 \mathrm{~ms}$ with vision (62\%), 2,058 impacts at $T=600 \mathrm{~ms}$ without vision $(29 \%)$, 6,695 impacts at $T=800 \mathrm{~ms}$ with vision $(62 \%), 1,891$ impacts at $T=800 \mathrm{~ms}$ without vision (18\%), 3,271 impacts at $T=1,000 \mathrm{~ms}$ with vision (39\%), and 839 impacts at $T=1,000 \mathrm{~ms}$ without vision (10\%). These cycles thus represent the steady-state behavior during successful completion of the task. Given the corresponding percentages, note that the task was more difficultly performed without vision, or at the slowest tempo.

The steady-state trajectories, depending on the tempo and the vision condition, were characterized by the following parameters: the angular position of the arm at impact $\theta_{i}$, the radial position of the puck at impact $r$, the smallest angle of the arm during the cycle $\theta_{m}$ and the largest angle of the arm during the cycle $\theta_{M}$ (see Fig. 2, right panel). The arm angular positions were measured with respect to the bisecting line of the wedge, i.e., the vertical axis of the movement plane.

To assess whether the arms maintained a pose interval during the cycle, we computed the activity period of the impacting arm as the percentage of the movement cycle during which the arm was actually moving, according to a combined velocity $\left(\dot{\theta}>5^{\circ} / \mathrm{s}\right)$ OR acceleration $\left(\ddot{\theta}>20^{\circ} / \mathrm{s}^{2}\right)$ criterion. For instance, if this ratio equals $90 \%$ for a cycle, it means that the arm velocity and acceleration were simultaneously below $5 \%$ and $20 \% \mathrm{~s}^{2}$, respectively,-i.e., the arm was at rest-during $10 \%$ of the cycle. We also computed the synchronization between both arms during one cycle as their averaged relative phase, via the non-normalized correlation coefficient of both arms velocity $\dot{\theta}_{1}$ and $\dot{\theta}_{2}$ :

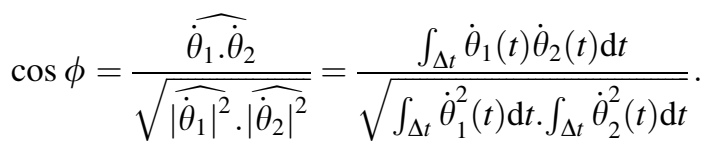

Synchronized in-phase or anti-phase movements lead to $\cos \phi=-1$ and $\cos \phi=1$, respectively. Decoupled movements, i.e., if at least one arm is at rest $(\dot{\theta}=0)$ at any time, lead to $\cos \phi=0$. All the parameters characterizing the arms trajectory were calculated for each flight time or arm cycle individually. The mean and standard deviation were calculated within each 2-min block, and then averaged across sessions and subjects.

Closed-loop control of the puck trajectory was analyzed by linear correlations of one perceived candidate variable (input of the controller) and one controlled variable (output of the controller). Significant correlations have been found between the puck energy during flight $\xi[k]$ (computed at mid-flight, since the puck flights were almost frictionless) and the arm velocity at the next impact $\dot{\theta}_{i}[k+1]$ (which is the most straightforward controlled variable). The puck energy was chosen as a potentially perceived and calculated state variable, since it depends only on the puck position and velocity which are two state variables measurable through oculomotor tracking (i.e., low position and velocity errors). The arm velocity at impact determines the energy restored to the puck (through the impact rule Eq. 1). Since the tempo of the task corresponds to one steady-state energy level of the puck for a fixed angular impact position (see Eq. 2), focusing on the energy control is a simple way to aggregate the influence of all the state variables in one single parameter to quantify how well the subjects controlled the task tempo. Differences between the experimental contexts will be discussed through the differences of both the correlation slopes (strength of the closed-loop tuning) and the correlation coefficients (variability). Both the puck energy $\xi$ and the arm velocity $\dot{\theta}_{i}$ were normalized w.r.t. the average values, in order to compare the correlations across different conditions corresponding to different averages. The parameters have been normalized block per block around the average of the block, i.e., $\bar{\xi}$ and $\overrightarrow{\dot{\theta}}_{i}$ :

$$
\begin{aligned}
\Delta \xi[k](\%) & =\frac{\xi[k]-\bar{\xi}}{\bar{\xi}} \times 100 \%, \\
\Delta \dot{\theta}_{i}[k](\%) & =\frac{\dot{\theta}_{i}[k]-\overline{\dot{\theta}}_{i}}{\overline{\dot{\theta}}_{i}} \times 100 \% .
\end{aligned}
$$

After normalization, we kept each impact as an individual event (point) to compute the averaged correlation slopes, across blocks, sessions and subjects. Note that the correlation slope must be negative if the task is stable, such that the deviations around the steady-state energy are compensated for by corrections of the arm velocity at impact. If $v_{n}^{-}$-hence the pre-impact energy $\xi[k]$-is smaller than steady-state, $\dot{\theta}_{i}[k+1]$ must be larger than steady-state in order to make $v_{n}^{+}$-hence the post-impact energy $\xi[k+1]$-closer to steady-state. The larger the absolute value of the slope, the more rapid this decay.

\section{Statistics}

The analysis of variance of the steady-state parameters was computed using classical factorial analysis of variance (ANOVAs). The first within-subject factor was the tempo $T$ and the second factor was the vision condition $(4 \times 2$ design). All ANOVAs were evaluated as significant for $P$-levels of 0.05 . Main and interaction effects were further analyzed using Tukey HSD post hoc tests. Negativity of the arm acceleration was further examined by single sample $t$-tests against zero, for the $4 \times 2$ conditions. 
Linear regressions were processed using a standard regression algorithm (least square minimization). Statistical comparisons were based on analysis of covariance (ANCOVA) that was applied to the normalized puck energy $\Delta \xi[k]$ at the mid-point of each flight, with the different conditions (tempo, vision) as factors and the normalized arm velocity at impact $\Delta \dot{\theta}_{i}[k+1]$ as co-variate (homogeneity of slope linear model).

All statistical analysis were completed using Statistica (StatSoft Inc., OK, USA).

\section{Results}

Steady-state trajectories

The subjects succeeded to juggle the puck close to the tempo $T$ dictated by the metronome, both with eyes open or closed (see Table 1).

The steady-state juggled pattern was a parabola, with radial impact position $r$ and angular aperture $2 \theta_{i}$. Figure 2 displays these steady-state trajectories, for the four tempi and the two vision conditions. The impact position of the arms $\theta_{i}$, the smallest angular position of the arms during cycle $\theta_{m}$, and the largest angular position of the arms during cycle $\theta_{M}$ are also represented. The numerical values of these parameters, and the position range $\left(\theta_{M}-\theta_{m}\right)$, are given in Table 1, pooled for both arms. Factorial ANOVAs revealed a strong significant dependence of all these variables except $r$ on the tempo (all $P<0.0001$ ). Accordingly, for $\Delta t, \theta_{i,} \theta_{m}$ and $\theta_{M}$, Tukey HSD post hoc tests revealed that any pair of corresponding to two different tempi were all significantly different from each other. For $r$, the eight values were not significantly different from each other. For the range $\left(\theta_{M}-\theta_{m}\right)$, Tukey test results are more tedious to report and do not seem to follow a clear trend across tempi. In contrast, only $\theta_{M}$ and the range $\left(\theta_{M}-\theta_{m}\right)$ significantly depended on the vision condition (both $P<0.0001$ ).
Tukey HSD post hoc tests here revealed that the pairwise vision conditions that were indeed significantly different from each other corresponded to $T=800$ and $1,000 \mathrm{~ms}$ (bold fonts on Table 1). The interaction between both factors was never significant. In order to increase (or decrease) the tempo, the subjects had the choice to reduce (or augment) either the radial position or the angular aperture (or the two of them, see Eq. 2). As reported in Table 1 and Fig. 2, the radial impact position did not significantly vary across conditions. In contrast, the dependence of the angular variables on the tempo suggests that the subjects adapted the aperture between the arms to juggle the different tempi: the faster the tempo, the smaller the aperture (Fig. 2).

Moreover, the dependence of $\theta_{M}$ and the range $\left(\theta_{M}-\right.$ $\left.\theta_{m}\right)$ on the vision condition suggests that the subjects actuated their arms over a smaller position range with vision than without, the difference reaching significance for the two slowest tempi.

\section{Rhythmic or discrete behavior}

In normal visual condition, two stereotyped actuation profiles were adopted by the subjects. The fastest tempi (e.g., $T=400 \mathrm{~ms}$, see Fig. 3, left) were characterized by rhythmic and sustained actuation of both arms in synchrony (see velocity profiles), resembling to a sinusoidal harmonic movement. In contrast, for the slow tempi (e.g., $T=1,000 \mathrm{~ms}$, see Fig. 3, right), the arms were actuated one after the other to impact the puck. In this case, the actuation was a train of discrete movements.

As illustrated by these typical cycles, the synchronization between the arms and the activity period (see "Materials and methods") strongly depended on the task tempo. The transition from rhythmic and synchronized movements (fast tempo) to discrete and decoupled movements (slow tempo) is clearly illustrated in Fig. 4 which

Table 1 The table reports the means of the average and standard deviation of the flight time $\Delta t$, the angular position at impact $\theta_{i}$, the radial position of the puck at impact $r$, the smallest angular position of the arm during the cycle $\theta_{m}$, the largest angular position of the arm during the cycle $\theta_{M}$, and the arm position range $\theta_{M}-\theta_{m}$

\begin{tabular}{|c|c|c|c|c|c|c|c|c|}
\hline \multirow{2}{*}{$\begin{array}{l}\text { Tempo } T(\mathrm{~ms}) \\
\text { Vision }\end{array}$} & \multicolumn{2}{|l|}{400} & \multicolumn{2}{|l|}{600} & \multicolumn{2}{|l|}{800} & \multicolumn{2}{|l|}{1,000} \\
\hline & With & Without & With & Without & With & Without & With & Without \\
\hline$\Delta t(\mathrm{~ms})$ & $415 \pm 63$ & $409 \pm 60$ & $601 \pm 93$ & $602 \pm 89$ & $808 \pm 132$ & $817 \pm 132$ & $1,000 \pm 173$ & $992 \pm 154$ \\
\hline$\theta_{i}(\mathrm{deg})$ & $22.0 \pm 4.8$ & $22.3 \pm 4.5$ & $32.1 \pm 5.0$ & $32.5 \pm 5.5$ & $42.6 \pm 5.2$ & $42.2 \pm 6.5$ & $51.0 \pm 5.7$ & $49.9 \pm 6.6$ \\
\hline$r(\mathrm{~mm})$ & $620 \pm 109$ & $611 \pm 145$ & $591 \pm 108$ & $577 \pm 151$ & $595 \pm 122$ & $600 \pm 161$ & $594 \pm 143$ & $600 \pm 162$ \\
\hline$\theta_{m}(\operatorname{deg})$ & $17.3 \pm 4.4$ & $17.7 \pm 3.9$ & $26.0 \pm 4.5$ & $26.6 \pm 4.7$ & $35.7 \pm 5.1$ & $35.0 \pm 5.1$ & $41.4 \pm 6.6$ & $41.4 \pm 5.4$ \\
\hline$\theta_{M}(\operatorname{deg})$ & $28.7 \pm 5.5$ & $29.5 \pm 4.7$ & $40.3 \pm 5.7$ & $43.8 \pm 5.5$ & $52.0 \pm 6.1$ & $55.2 \pm 5.7$ & $61.9 \pm 6.8$ & $66.2 \pm 5.7$ \\
\hline$\theta_{M}-\theta_{m}(\operatorname{deg})$ & $11.4 \pm 3.7$ & $11.8 \pm 3.1$ & $14.3 \pm 4.6$ & $17.2 \pm 3.7$ & $16.3 \pm 5.4$ & $20.2 \pm 4.9$ & $20.5 \pm 7.9$ & $24.8 \pm 6.0$ \\
\hline
\end{tabular}

These variables are given for the four tempi and the two vision conditions. Tukey HSD post hoc tests revealing significant $(P<0.01)$ differences between the two vision conditions are highlighted in bold characters 
$400 \mathrm{~ms}$

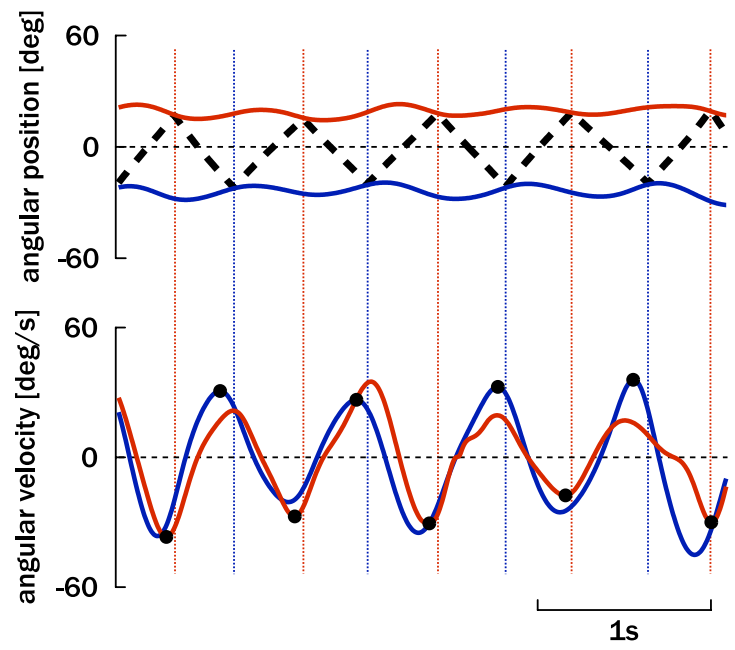

Fig. 3 Typical angular trajectories at fast tempo $(T=400 \mathrm{~ms}$, left $)$ and slow tempo $(T=1,000 \mathrm{~ms}$, right $)$. Top panels, the right and left arm angular position (red and blue, respectively) and the angular position of the puck (dashed black, see the right panel with color legend) are represented. For clarity, the radial position of the puck is

reports the synchronization index and the activity period for the $4 \times 2$ conditions. Factorial ANOVAs revealed that both variables depended on the tempo [both $F(3,399)>38$, $P<0.0001$ ], and on the vision condition [both $F(1,399)>10, P<0.05]$, but not on their interaction. In sum, Fig. 4 shows that the faster the tempo, the larger the synchronization index and the longer the activity period of the arms. At the fastest tempo $(T=400 \mathrm{~ms})$, both arms were almost always moving (activity period close to $100 \%$ )
$1000 \mathrm{~ms}$

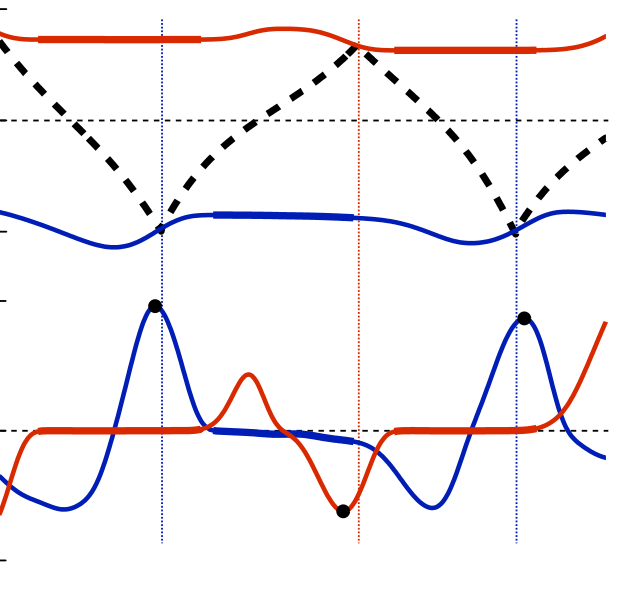

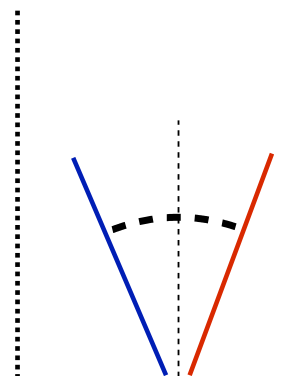

Fig. 4 The left panels depict the mean of the synchronization index (cosine of the average relative phase, see "Materials and methods"- top) and of the activity period (bottom) of the arms, as a function of the tempo $T$ and the vision condition: with vision (black) and without vision (gray). Error bars denote \pm 0.95 Conf. Interval. The right panels depict the standard deviations of the same variables not represented in this graph. The thicker portions emphasize the periods when the arms are at rest. The vertical lines denote the impact times. Bottom panels, the right and left arm velocity (red and blue, respectively) are represented. The black dots denote the point of maximum velocity (velocity peak) around impacts

in synchrony (synchronization index close to 1). As the tempo decreased, the arms were actuated more and more intermittently, resulting in a loss in synchrony and a decreased activity period.

Moreover, it shows the same trend both with and without vision. It further reveals that the absence of vision favored the sustained and synchronized actuation mode. The smallest difference is observed at $T=400 \mathrm{~ms}$ since, in this case, the actuation was rhythmic and synchronized regardless of the
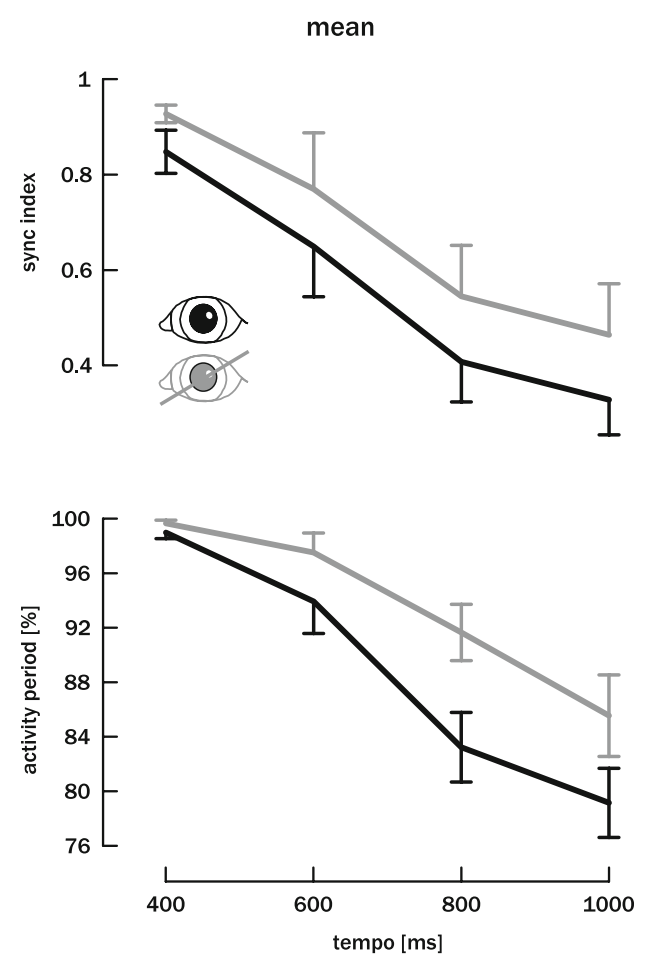

standard deviation
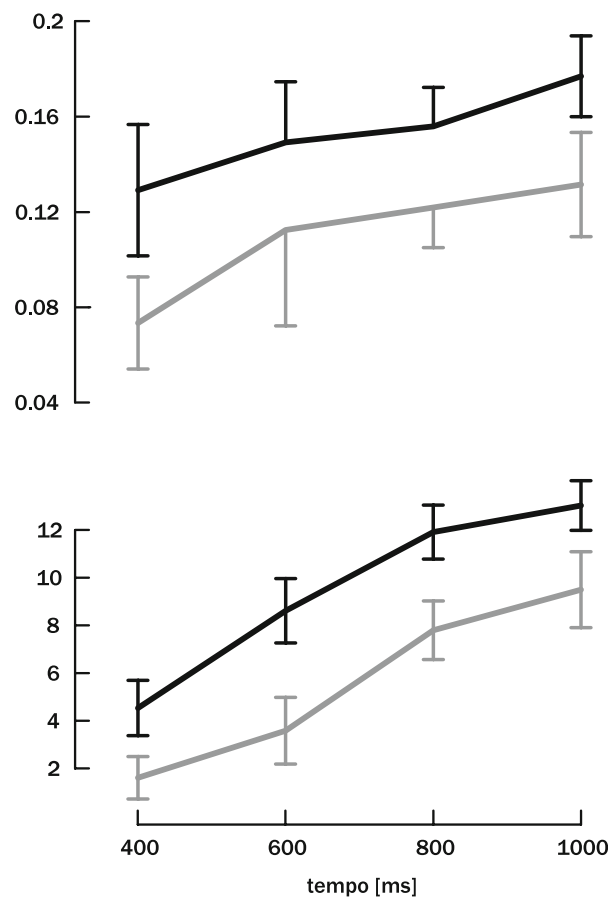
vision condition. Figure 4 also reports the standard deviation analysis. Factorial ANOVAs also revealed that the standard deviation of both variables depended on the tempo [both $F(3,399)>7, P<0.0001]$, and on the vision condition [both $F(1,399)>28, P<0.0001$ ], but not on their interaction. The variability of the bimanual coordination indices increased as the tempo decreased and-unexpectedlydecreased without visual information. In sum, the more discrete the control, the more variable the bimanual actuation profile. In contrast, rhythmicity implied more consistency (less variability in arms trajectory).

\section{Arm acceleration at impact}

In our results, the arm position and velocity at impact did not vary significantly across the two vision conditions: their steady-state values are fixed by the period-one orbit, i.e., the reference tempo and the coefficient of restitution (Ronsse et al. 2006).

In contrast, as illustrated on Fig. 5, the arm acceleration at impact varied across conditions. Factorial ANOVA revealed a dependence on the vision condition $[F(1,399)=34.3$, $P<0.0001]$, and on the tempo $[F(3,399)=3, P<0.05]$, while the dependence on their interaction was not significant. Tukey HSD post hoc tests further revealed that the pairwise differences between the data with and without vision were significant for any given tempo $T(P<0.05)$, but for $T=400 \mathrm{~ms}$. The standard deviations ranged around $220 \mathrm{deg} / \mathrm{s}^{2}$ and did not reach significant dependence on the factors or their interaction. With vision at slower tempi, i.e., at $T=600,800$ and $1,000 \mathrm{~ms}$, the acceleration at impact was not significantly different from zero (single sample $t$-tests, all $P>0.59$ ). The five other conditions corresponded to acceleration significantly different from zero and negative (all $P<0.01$ ). In sum, the subjects adopted more negative

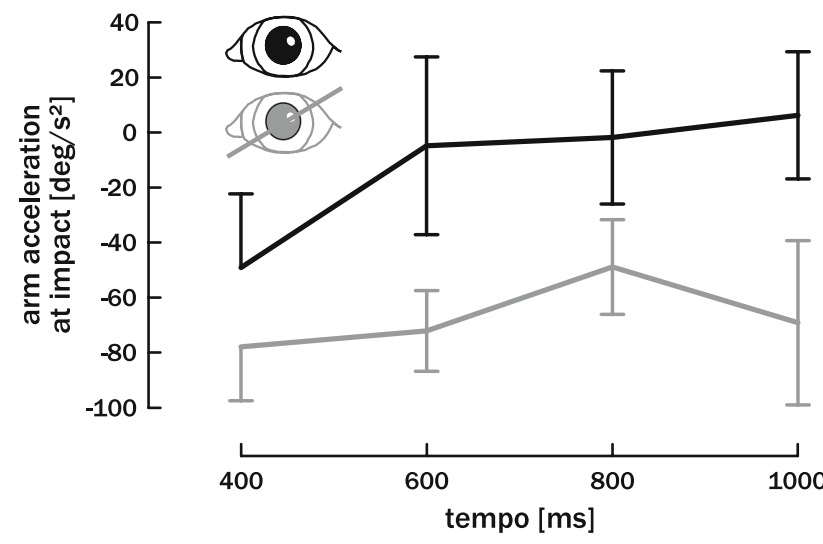

Fig. 5 Mean of the acceleration of the arm at impact, as a function of the tempo $T$ and the vision condition: with vision (black) and without vision (gray). Error bars denote \pm 0.95 Conf. Interval acceleration at impact without visual information (significant for all but for $T=400 \mathrm{~ms}$ ).

Average energy of the arm

As suggested by Fig. 3, bottom, the two identified actuation modes (rhythmic or discrete) corresponded to different velocity profiles. We analyzed whether this difference may also reflect a difference in the energy of the arms during the movement execution, assuming that larger energy could require a larger effort to impact the puck at the proper velocity. Energy can thus be a way to quantify to control efficiency. We calculated an indirect estimation of this quantity via the average absolute velocity of the impacting arm during one cycle, since the arm kinetic energy is directly proportional to the square of the velocity (potential energy has been neglected).

This quantity is displayed on Fig. 6. The influence of the tempo $[F(3,399)=13.5]$ and the vision condition $[F(1,399)$ $=47.4$ were significant (factorial ANOVA, all $P<0.0001$ ), but not their interaction. As suggested by the confidence intervals, Tukey HSD post hoc tests revealed that the pairwise differences between the data with and without vision were significant for any given tempo $T$ $(P<0.05)$, but for $T=400 \mathrm{~ms}$. Figure 6 reveals that the absence of vision increased the average velocity at the four tested tempi. Once again, the smallest difference between vision conditions is reported at the fastest tempo ( $T=400 \mathrm{~ms}$ ). Moreover, with vision, the fastest tempo ( $T=400 \mathrm{~ms}$ ) corresponded to larger average velocity than the three other tempi (significant for $T=800,1,000 \mathrm{~ms}$, Tukey HSD post hoc tests, $P<0.0001)$. The standard deviations ranged around $7 \%$ s and did not reach significant dependence on the factors or their interaction.

In summary, the conditions corresponding to the rhythmic mode of control corresponded not only to the

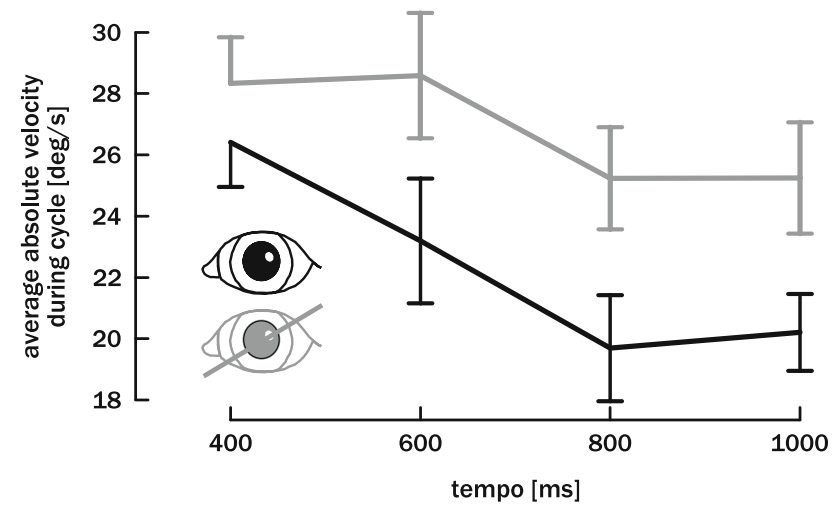

Fig. 6 Mean of the averaged absolute velocity of the impacting arm across a complete arm cycle, as a function of the tempo $T$ and the vision condition: with vision (black) and without vision (gray). Error bars denote \pm 0.95 Conf. Interval 
largest average velocities but also to the "more negative" acceleration at impact (see Fig. 5), hereby suggesting the recruitment of a control strategy that exploits the task robustness (negative acceleration), sacrificing the efficiency (energy).

\section{Control of the puck energy}

To assess potential contributions of active control based on perceived errors, we investigate in the present section how the impact velocity of the arm compensates for a mismatch between the puck energy during the preceding flight and its steady-state, depending on the requested tempo $T$ (Eq. 2). This correlation between the two variables must be negative (smaller puck energy requires larger impact velocity, and vice versa). In sum, the larger the slope in absolute value, the stronger the control, as mentioned in the "Materials and methods" section.

Figure 7 reveals that the slopes were negative with highly significant correlation coefficients $(P<0.0001)$ at the four tempi, with or without vision. Moreover, they were similar across tempi for a given vision condition: they ranged between -1 and -1.15 in the presence of visual information, while they were about twice smaller (between -0.49 and -0.59$)$ in the absence of visual information. The analysis of covariance revealed that the slope coefficients significantly depended on the vision condition
$[F(1,28160)=1,230.6]$, on the tempo $[F(3,28160)=12.1]$ (both $P<0.0001)$ and on their interaction $[F(3,28160)$ $=4.7, P<0.05]$. The large number of degrees of freedom is due to the fact that each impact was considered as an independent event for this analysis, such that the picture captures the global average of the subjects behavior. However, each point has been normalized with respect to its own block (see Eq. 3). It is further observed that the data variance was higher without vision (correlation coefficients around -0.47) than with vision (correlation coefficients around -0.66). This result is not in contradiction with the result reported on Fig. 4: this figure revealed that the arms trajectory was less variable without vision, while here we established that the puck energy was more variable without vision. These results are not incompatible: less variability in the puck energy could be achieved by a finer tuning of the arms, leading to more variability in their trajectory.

Since a larger slope should correspond to a better regulation of the energy around steady-state, we analyzed the variability of the energy around its steady-state for the 4 $\times 2$ conditions. Table 2 shows the mean and standard deviation of the puck energy at mid-flight, as a function of the tempo and vision condition. A factorial ANOVA revealed a small dependence of the mean energy on the tempo $[F(3,399)=6.3, P<0.05]$ but neither on the vision condition nor on their interaction. More interestingly, the same factorial ANOVA analysis of the standard deviation 400ms

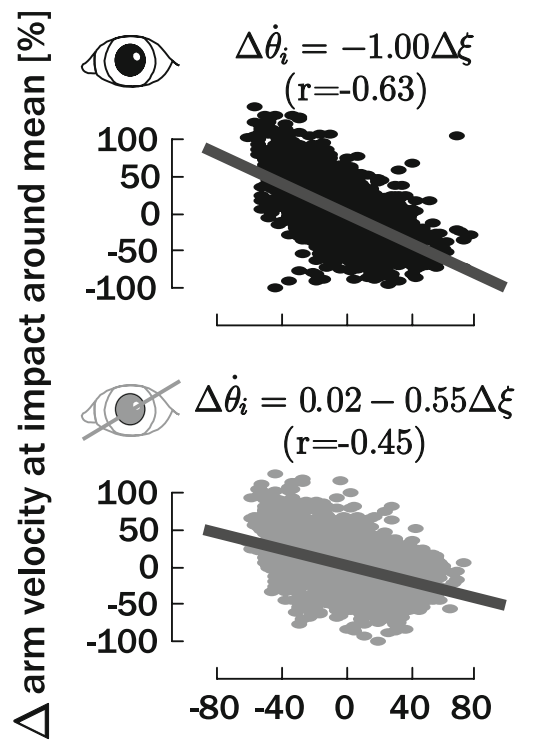

$600 \mathrm{~ms}$
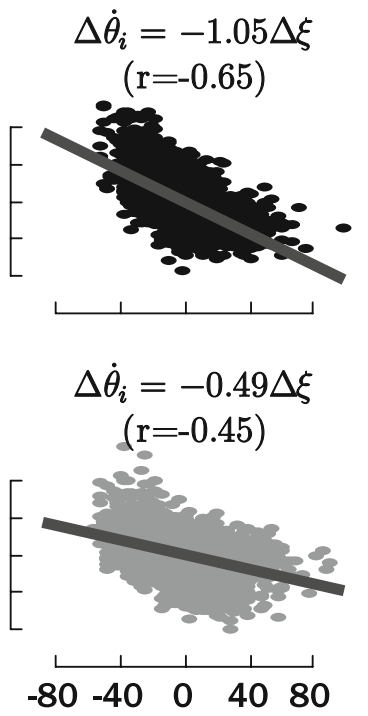

$800 \mathrm{~ms}$

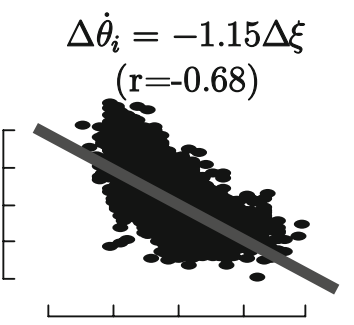

$\Delta \dot{\theta}_{i}=0.02-0.59 \Delta \xi$ $(\mathrm{r}=-0.51)$

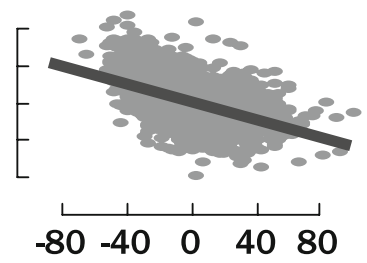

$1000 \mathrm{~ms}$

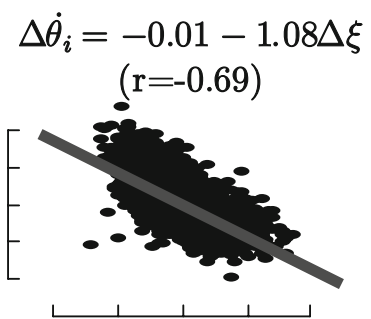

$\Delta \dot{\theta}_{i}=0.01-0.56 \Delta \xi$ $(\mathrm{r}=-0.48)$

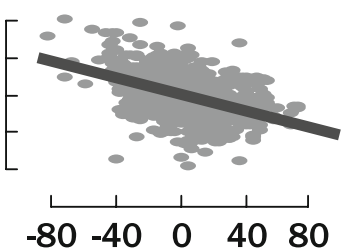

$\Delta$ energy at mid-flight around mean [\%]

Fig. 7 The relative $(\Delta)$ velocity of the arm at the impact $\dot{\theta}_{i}[k+1]$ as a function of the relative $(\Delta)$ energy of the puck at mid-flight $\xi[k]$ (around means), for each tempo and vision conditions. The black points (top) depict the impacts with vision and the gray points (bottom) those without vision. The straight lines represent the linear regressions of these data, the regression slopes and the correlation coefficients being indicated above each graph $(P<0.0001$ in all conditions) 
Table 2 The table reports the mean and standard deviation (SD) of the puck energy (per unit of mass) at mid-flight $\left(10^{6} \mathrm{~m}^{2} / \mathrm{s}^{2}\right)$, depending on the tempo and vision condition

\begin{tabular}{lllll}
\hline $\begin{array}{l}\text { Tempo } \\
T \text { (ms) }\end{array}$ & 400 & 600 & 800 & 1,000 \\
\hline With vision & $2.03 \pm 0.36$ & $1.91 \pm 0.31$ & $1.91 \pm 0.33$ & $1.92 \pm 0.36$ \\
$\begin{array}{l}\text { Without } \\
\text { vision }\end{array}$ & $2.02 \pm 0.48$ & $1.87 \pm 0.45$ & $1.94 \pm 0.44$ & $1.96 \pm 0.42$ \\
\hline
\end{tabular}

of the energy revealed dependences on the vision condition $[F(1,399)=168.4, P<0.0001]$, on the tempo $[F(3,399)$ $=3.8, P<0.05]$ and on the interaction between both factors $[F(3,399)=4.8, P<0.05]$. In sum, the variance of the data was much larger without than with visual information. The subjects maintained the puck energy more efficiently around its steady-state value with vision, and this corresponded to larger slopes in the correlations reported in Fig. 7.

\section{Discussion}

The present paper investigates an original rhythmic task, requiring the stabilization of a juggled puck through an appropriate bimanual actuation pattern. The impact task is a 2D bimanual extension of the classical movement of a ball bouncing on a racket (Schaal et al. 1996; Sternad 1999; Sternad et al. 2001a, b; Katsumata et al. 2003; deRugy et al. 2003; Dijkstra et al. 2004; Wei et al. 2007). The task was performed at four different tempi, and in two distinct vision conditions.

The task context constrains the actuation strategy. First, the withdrawal of visual information deprives the subject from a major source of sensory inflow, and most likely deteriorates the quality of the feedback estimation of the state of the body (arm kinematics) and the environment (puck trajectory). Second, increasing the tempo reduces the time available to plan and execute each movement individually. We showed that the experimental conditions influence the control strategy, despite an unchanged task objective.

Two different primitives of control?

Our analysis revealed that different strategies were adopted for the execution of the arms movements, depending on the context of the task, i.e., the requested tempo and the presence or absence of vision. A rhythmic actuation mode — similar to a sinusoidal harmonic movement—was favored not only at the fastest tempi, but also by suppressing the visual information, whatever the tempo.
The increase in the activity period was correlated with an increase in the bimanual synchronization index, in agreement with basic intrinsic bimanual coordination rules (see, e.g., Swinnen 2002; Swinnen and Wenderoth 2004, for reviews). In contrast, with vision at the slowest tempi, the actuation was a train of discrete movements (Hogan and Sternad 2007). It is important to notice that the change in actuation mode was not due to mechanical limitations of the setup, since the average maximal angular position of the arms $\left(\theta_{M}\right.$ always $\left.<67^{\circ}\right)$ remained far from the table frame (i.e., $\pm 90^{\circ}$ ) even at the slowest tempo (see Fig. 2; Table 1). The dynamical consequences of these different execution strategies are explored in the rest of this discussion, in the light of the trade-off between efficiency and robustness.

The rhythmic actuation mode-adopted both at the fastest tempi and without vision-highlights an interesting property of the impact model. Indeed this actuation mode corresponds to negative acceleration at impact. Negative acceleration at impact has been demonstrated to be a necessary condition for the stability of passive strategies in impact tasks (Schaal et al. 1996; Sternad 1999; Sternad et al. 2001a, b; deRugy et al. 2003), hence suggesting that the sustained actuation mode was recruited to reduce the need for feedback processing. Consistently, this strategy was adopted when either the sensory inflows were altered (withdrawal of visual information) or when the time available for sensory integration, planning and execution of individual movements was the shortest (fastest tempo), that is, in contexts exposing the robustness of the actuation. Interestingly, we have demonstrated that negative impact acceleration can directly quantify the closed-loop robustness with a simple mechanical model of this task (Ronsse et al. 2007). Note that we provided convincing results that only negative impact acceleration permits to juggle the puck either without (Ronsse et al. 2006) or with limited feedback (Ronsse et al. 2007) in robotics experiments.

In contrast, when the tempo was slower and the visual information was available, the acceleration was close to zero, and the subjects preferred to rest on the discrete mode of control. Nearly zero arm acceleration at impact means that the maximum velocity of the arm during the cycle (the velocity peak) is very close to the velocity at impact. This can be a signature of a strategy reducing the energy cost of the movement. Indeed, since the impact velocity is constrained by the mechanics of the setup (i.e., the energy to restore to the puck, see Eq. 1), the cheapest movement to reach this velocity necessitates to impact at the velocity peak. If the velocity peak were at impact, the arm would never move at a larger velocity that the one requested by the impact rule. The discrete actuation mode thus reduces the energy of the movement around impacts. Moreover, long periods of inactivity between the impacts were 
detected in that mode, which further contributes to reduce the energy cost of the movement. Finally, the average absolute velocity throughout the whole arm cycle was smaller than in the other conditions. Consistently, Table 1 also revealed that the position range of the arms,-i.e., the movement amplitude $\theta_{M}-\theta_{m}$-within cycles was systematically larger without than with vision, while the impact position did not significantly change. In summary, both the average velocity and the position range were larger when the execution mode was more rhythmic and synchronized between both arms. An increased energy expense (reducing the efficiency in energy cost) was the price to pay for a more negative acceleration at impact (increasing the robustness).

It has been suggested that human movements are programmed by proper combinations of units of action-or primitives-(see, e.g., Hogan and Sternad 2007, for a recent review), both in the combination of several submovements into a single movement (Novak et al. 2002), and in the combination of discrete and rhythmic movements (Ijspeert et al. 2003). Supporting this last assumption, Schaal et al. (2004) demonstrated in an imaging study that discrete and rhythmic movements are executed by different cortical and cerebellar structures. The present study also illustrates that a rhythmic mode or a train of discrete actuation mode are recruited within a single task, just depending on the context, i.e., the tempo and the available feedback; and that, consistently with Schaal et al. (2004), the rhythmic mode may be controlled by a simpler computational structure than the discrete mode, since it is based on a reduced need of feedback (better robustness).

Active control of the puck energy

Our data suggest also a feedback correction of the arm impact velocity between successive impacts, primarily correlated to the energy of the puck during the preceding flight. The error feedback gain was significantly negative for the eight conditions, and twice larger with than without vision. For the conditions corresponding to negative acceleration, this negative acceleration has naturally contributed to make the gain negative, i.e., independently of the presence of sensory inflows: larger energy during flight normally corresponds to longer flight time, thus to smaller impact velocity if the acceleration is negative. However, we checked by mean of numerical simulations of the task model that sensorless stabilization of the puck (under sinusoidal actuation of the arms, thus with negative acceleration at impact) does generate negative slopes but with smaller magnitudes than the slopes reported here (data not shown). In sum, even with negative acceleration, the correlation between the energy during flights and the arm velocity at the next impact is - at least partly-a basic manifestation of closed-loop control in our data, whose tuning reveals also the trade-off between efficiency and robustness. Indeed, a primary role of any feedback loop is to reduce the sensitivity to uncertainties in the plant model: a higher feedback gain improves the closed-loop performance (a twice larger gain in the presence of vision resulted in less variability of the puck energy around the steady-state) but deteriorates its robustness, increasing the proneness to instability in the presence of delays or noise measurement (i.e., if the sensors are unperfect, see, e.g., Franklin et al. 2005; Åström and Murray 2008; Boulet and Duan 2007). In summary, it is better to rely on feedback active control when the sensory inflows are accurate, while it is better to rely on passive control (i.e., control signals not depending on the sensory inflows) when they are noisy. In general, the variability around the correlation slopes remained important, since the puck energy is potentially difficult to be accurately estimated, while other closed-loop mechanisms may have been implemented by the subjects and not captured by this simple model.

With vision (and at comfortable tempo), the subjects could use it to estimate the puck energy (e.g., at mid-flight). Huys and Beek (2002) reported interesting results on the gaze dynamics in the three-balls cascade juggling (one of the most classical juggling pattern): the point-of gaze was confined close to the zenith of the balls trajectory, while the gaze and balls dynamics were frequency-locked, suggesting both small position and velocity errors around zenith. Consistently, the puck energy (depending on both the position and the velocity) could be potentially estimated (at mid-flight) in this kind of juggling task. Consequently, less noisy estimation of the puck energy would result in higher feedback gain. In our data, the gain of the feedback was not affected by the tempo in a given visual condition, while the fastest tempo required to process the sensory feedback and update the corresponding movement in a very short time. This could be another consequence of having adopted the rhythmic mode of control (with negative acceleration) as the tempo increased, since negative acceleration naturally contributes to make the gain negative without sensory processing (see above). This pleads once again in favor of adopting the robust strategy with negative acceleration as the tempo increases.

The rhythmic actuation mode exploits valuable dynamical properties of impact tasks, in order to make the control robust. This actuation mode is likely implemented from a baseline oscillation whose amplitude and/or phase is controlled on the basis of the sensory inflows. A similar actuation mode is described in the analysis of locomotion, i.e., a rhythmic movement in which the visual inflow is not permanently processed and where passive dynamical properties are of prime interest (see, e.g., McGeer 1990; 
Goswami et al. 1998; Collins et al. 2001, 2005). Interestingly, a control structure based on closed-loop control of amplitude and phase has been proposed by Buschges (2005) for the control of locomotory central pattern generators. Moreover, Kuo (2002) discussed the fundamental properties of the trade-off between efficiency and robustness for a combined feedback-feedforward model of a central pattern generator for locomotion. Kuo's main conclusion was that a purely feedforward central pattern generator is highly sensitive to unexpected disturbances. In contrast, a pure feedback control analogous to reflex pathways can compensate for disturbances, but is poorly robust to imperfect sensors. He illustrated through an elegant model that the best trade-off results in a proper combination of feedback and feedforward.

In contrast, the discrete mode is easily viewed as a train of individual movements, executed under optimality principles minimizing the energy cost and controlled to reach the desired velocity at the expected impact time (Land and McLeod 2000; Ronsse et al. 2007). The execution of these movements could be modeled and quantified by the theory of optimal control (Bryson and Ho 1969) which has been successful in modeling typical discrete movements like reaching, pointing or aiming (Todorov and Jordan 2002; Scott 2004; Todorov 2004, 2006; Bays and Wolpert 2007; Liu and Todorov 2007).

The MOSAIC model proposed by Wolpert and colleagues (Wolpert and Kawato 1998; Jordan and Wolpert 1999; Wolpert and Ghahramani 2000; Haruno et al. 2001) is a general framework to model the ability to generate accurate and appropriate motor behavior under many different and often uncertain environmental and contextual conditions. This architecture has been proposed for motor control, on the basis of multiple pairs of forward (predictor) and inverse (controller) models stored in the brain. A similar architecture could be elaborated for our task, in which the pairs of internal models (for control) are different for the two primitives of fundamental behavior (rhythmic-discrete), and may help to program accurate movements despite delays in the loop (Desmurget and Grafton 2000).

This paper main objective was to illustrate the importance of an essential concept of control theory,-i.e., the robustness of the actuation strategy-in the execution of a motor control task. Through an informative example, we illustrated that the control behavior may balance differently the robustness and the efficiency with respect to dynamical criteria, while the task objective remained unchanged.

Acknowledgments The authors would like to thank the anonymous reviewers for their helpful comments that improved the paper. Grants: This paper presents research results of the Belgian Network DYSCO
(Dynamical Systems, Control, and Optimization), funded by the Interuniversity Attraction Poles Programme, initiated by the Belgian State, Science Policy Office. The scientific responsibility rests with its authors. PL was funded by the "Fonds National de la Recherche Scientifique"; the "Fondation pour la Recherche Scientifique Médicale"; and an internal research grant ("Fonds Spéciaux de Recherche") from the Université catholique de Louvain. JLT and PL were funded by the European Space Agency (EU) and Prodex (Belgium).

\section{References}

Åström KJ, Murray RM (2008) Feedback systems. An introduction for scientists and engineers. Princeton University Press, New Jersey

Bays PM, Wolpert DM (2007) Computational principles of sensorimotor control that minimize uncertainty and variability. J Physiol 578(Pt 2):387-396

Boulet B, Duan Y (2007) The fundamental tradeoff between performance and robustness-a new perspective on loop shaping. IEEE Control Syst Mag 27(3):30-44

Bryson AE, Ho YC (1969) Applied optimal control: optimization, estimation, and control. Blaisdell, Waltham

Buschges A (2005) Sensory control and organization of neural networks mediating coordination of multisegmental organs for locomotion. J Neurophysiol 93(3):1127-1135

Clarke AH, Ditterich J, Druen K, Schonfeld U, Steineke C (2002) Using high frame rate cmos sensors for three-dimensional eye tracking. Behav Res Methods Instrum Comput 34(4):549 560

Collins SH, Wisse M, Ruina A (2001) A three-dimensional passivedynamic walking robot with two legs and knees. Int J Robot Res 20(7):607-615

Collins S, Ruina A, Tedrake R, Wisse M (2005) Efficient bipedal robots based on passive-dynamic walkers. Science 307(5712):1082-1085

Desmurget M, Grafton S (2000) Forward modeling allows feedback control for fast reaching movements. Trends Cogn Sci 4(11):423-431

de Rugy A, Wei K, Muller H, Sternad D (2003) Actively tracking 'passive' stability in a ball bouncing task. Brain Res 982(1):6478

Dijkstra TMH, Katsumata H, de Rugy A, Sternad D (2004) The dialogue between data and model: Passive stability and relaxation behavior in a ball bouncing task. Nonlinear Stud 11(3):319-344

Franklin G, Powell JD, Emami-Naeini A (2005) Feedback control of dynamic systems, 5th edn. Prentice Hall, Englewood Cliffs

Goswami A, Thuilot B, Espiau B (1998) A study of the passive gait of a compass-like biped robot: symmetry and chaos. Int $\mathrm{J}$ Robot Res 17(12):1282-1301

Harris CM, Wolpert DM (1998) Signal-dependent noise determines motor planning. Nature 394(6695):780-784. http://dx.doi.org/10.1038/29528

Harris CM, Wolpert DM (2006) The main sequence of saccades optimizes speed-accuracy trade-off. Biol Cybern 95(1):21-29. http://dx.doi.org/10.1007/s00422-006-0064-x

Haruno M, Wolpert DM, Kawato M, (2001) Mosaic model for sensorimotor learning and control. Neural Comput 13(10):22012220

Hogan N, Sternad D (2007) On rhythmic and discrete movements: reflections, definitions and implications for motor control. Exp Brain Res 181(1):13-30 
Huys R, Beek PJ (2002) The coupling between point-of-gaze and ball movements in three-ball cascade juggling: the effects of expertise, pattern and tempo. J Sports Sci 20(3):171-186

Ijspeert A, Nakanishi J, Schaal S (2003) Learning attractor landscapes for learning motor primitives. In: Advances in neural information processing systems, vol 15. MIT Press, Cambridge, pp $1547-1554$

Jordan MI, Wolpert DM (1999) Computational motor control. In: Gazzaniga M (ed) The cognitive neurosciences. MIT Press, Cambridge

Karniel A, Inbar GF (2000) Human motor control: Learning to control a time-varying, nonlinear, many-to-one system. IEEE Trans Syst Man Cybern C Appl Rev 30(1):1-11

Katsumata H, Zatsiorsky V, Sternad D (2003) Control of ball-racket interactions in rhythmic propulsion of elastic and non-elastic balls. Exp Brain Res 149(1):17-29

Kelso JAS (1995) Dynamic patterns. The self-organization of brain and behavior. MIT Press, Cambridge

Kelso JAS, Southard DL, Goodman D (1979) On the nature of human interlimb coordination. Science 203(4384):1029-1031

Kuo AD (2002) The relative roles of feedforward and feedback in the control of rhythmic movements. Motor Control 6(2):129-145

Land MF, McLeod P (2000) From eye movements to actions: how batsmen hit the ball. Nat Neurosci 3(12):1340-1345

Liu D, Todorov E (2007) Evidence for the flexible sensorimotor strategies predicted by optimal feedback control. J Neurosci 27(35):9354-9368. http://dx.doi.org/10.1523/JNEUROSCI. 1110-06.2007

McGeer T (1990) Passive dynamic walking. Int J Robot Res 9(2):62-82

Nakano E, Imamizu H, Osu R, Uno Y, Gomi H, Yoshioka T, Kawato M (1999) Quantitative examinations of internal representations for arm trajectory planning: minimum commanded torque change model. J Neurophysiol 81(5):2140-2155

Novak KE, Miller LE, Houk JC (2002) The use of overlapping submovements in the control of rapid hand movements. Exp Brain Res 144(3):351-364

Ronsse R, Lefevre P, Sepulchre R (2006) Sensorless stabilization of bounce juggling. IEEE Trans Robot 22(1):147-159

Ronsse R, Lefevre P, Sepulchre R (2007) Rhythmic feedback control of a blind planar juggler. IEEE Trans Robot 23(4):790-802
Ronsse R, Lefevre P, Sepulchre R (2008) Robotics and neuroscience: a rhythmic interaction. Neural Netw (accepted for publication)

Schaal S, Schweighofer N (2005) Computational motor control in humans and robots. Curr Opin Neurobiol 15(6):675-682

Schaal S, Atkeson CG, Sternad D (1996) One-handed juggling: a dynamical approach to a rhythmic movement task. J Mot Behav 28(2): 165-183

Schaal S, Sternad D, Osu R, Kawato M (2004) Rhythmic arm movement is not discrete. Nat Neurosci 7(10):1136-1143

Scott SH (2004) Optimal feedback control and the neural basis of volitional motor control. Nat Rev Neurosci 5(7):532-546

Sternad D (1999) Juggling and bouncing balls: parallels and differences in dynamic concepts and tools. Int J Sport Psychol 30(4):462-489

Sternad D, Duarte M, Katsumata H, Schaal S (2001a) Bouncing a ball: tuning into dynamic stability. J Exp Psychol Hum Percept Perform 27(5):1163-1184

Sternad D, Duarte M, Katsumata H, Schaal S (2001b) Dynamics of a bouncing ball in human performance. Phys Rev E 6301(1), art. no. 011902

Swinnen SP (2002) Intermanual coordination: from behavioural principles to neural-network interactions. Nat Rev Neurosci 3(5):348-359

Swinnen SP, Wenderoth N (2004) Two hands, one brain: cognitive neuroscience of bimanual skill. Trends Cogn Sci 8(1):18-25

Todorov E (2004) Optimality principles in sensorimotor control. Nat Neurosci 7(9):907-915

Todorov E (2006) Optimal control theory. In: Doya K, Ishii S, Pouget A, Rao RP (eds) Bayesian brain. MIT Press, Cambridge

Todorov E, Jordan MI (2002) Optimal feedback control as a theory of motor coordination. Nat Neurosci 5(11):1226-1235

Wei K, Dijkstra TMH, Sternad D (2007) Passive stability and active control in a rhythmic task. J Neurophysiol 98:2633-2646. http://dx.doi.org/10.1152/jn.00742.2007

Wolpert DM, Ghahramani Z (2000) Computational principles of movement neuroscience. Nat Neurosci 3(Suppl):1212-1217

Wolpert DM, Kawato M (1998) Multiple paired forward and inverse models for motor control. Neural Netw 11(7-8):1317-1329 